\title{
Zwei Briefe aus den Tagen der lutherischen Orthodoxie.
}

\author{
Von G. Kawerau.
}

Die beiden nachfolgend zum Abdruck gelangenden Schreiben sind mir ans Privatbesitz in Frankfurt a. M. (Herrn Dr. med. Nebel) freundlicbst mitgeteilt worden. Ersteres fuhrt in die Zeit karz vor Pablikation der Konkordienformel, und zeigt, wie die Bemulhnngen des Kurfursten Angust, Landgraf Wilhelm von Hessen fur sein Konkordienwerk zo gewinnen, bis zur letzten Stande, freilich vergeblich, fortgesetzt wurden. Den völligen MiBerfolg versübt er sich und dem bekanntlich schlieBlich noch gewonnenen Pfalzgrafen Ludwig mit allgemeinen Zukunftshoffnungen, die wohl kaum so zarersichtlich gehegt wurden, wie die Worte klingen.

Das andere Schreiben fuhrt uns an das Ende des zweiten kryptocalvinistischen Streites und meldet die "Bekehrung“ des gefangengenommenen Hofpredigers Steinbach rom Calvinismus zur Iatherischen Sakramentslehre, ein charakteristisches Dokument jener Betrachtangsweise, welche die Hinwendang zar orthodoxen Lehre mit der Rettang und Umkehr des verlorenen Sohnes gleichsetzen konnte.

28. März 1580.

Kurfurst August and Karfurst Johann Georg an Pfalzgraf Ludwig.

Dem hochgeborenen fursten, herrn Ladwigen Pfaltzgrauen bei Rhein, des heiligen Römischen Reichs Ertztruchsessen vad Charfursten, Hertzogen in Baiern, vnserm freundlichen lieben rettern, ohmen, schwagern, brudern vad sohn.

Vnser freundlich dienst vnd was wir mehr liebes vnd gutes vermogen zanorn. Hochgeborner furst, freandlicher lieber vetter, oheim, schwager, brader vnd sohn. Beinerwart rberschicken wir E. L. Landgraff Wilhelms zu Hessen etc. wiederschreiben in Originali, darinnen S. L. vns allerseits auf vnser an dieselbe jungst ansgegangne vermahnunge des christlichen Concordien Buchs halben beantwortet, Vnd nach 
dem rus dem Churfürsten zu Sachssen dasselbe durch vnsern reitenden postboten erstlich zabracht, so baben wir es zu gewinnunge der zeit eröfnet rnd bitten sonders vleiß, E. I. wolle solches nicht vnfrenndlich vermercken, Vnd dieweil daraus zubefinden, das des Landgrauen L. auf voriger ihrer meinunge beruhet, vad von vns allerseits bißhero bei $\mathrm{S}$.L. alles das gathertziger wolmeinunge gethan, furgewant vad versucht worden, was disfals immer zathun möglich, also das wir nicht wissen, was nunmehr ferner oder anders zu than sein möchte, So mussen wir es dabei bleiben vnd berahen lassen, vnd achten, das S. L. halben die Publication des christlichen Concordien Buchs, darauf so viel fromme christliche hertzen mit seenlichem verlangen vod senftzen warten, nicht lenger eingestalt werden kan, wie wir dan E. L. noch vnlangst mit mehrerem ausfuhrlich zageschrieben, Vnd wollen zu Gott dem almechtigen hoffen, Wan die Formul der christlichen Concordien pablicirt, es werde nicht alleine durch seine gotliche verleihunge des Landgrauen za Hessen $L$. sondern noch viel andere, rnd anch ein guter theil von denen, so itzo noch dem Babstischen irthumben anhengigk vnd zagethan, za solchem christlichen wergk sich nähen rnd finden, vnd dadurch vieler einfaltiger frommer leute seelen heil vnd seligkeit gefördert vnd vortgesatzt werden, Wolten wir E. L. freandlich nicht verhalten, Vnd seint derselben jder zeit freundlich zu dienen willigk. Datum den xxviij. Martij anuo etc. Lxxx.

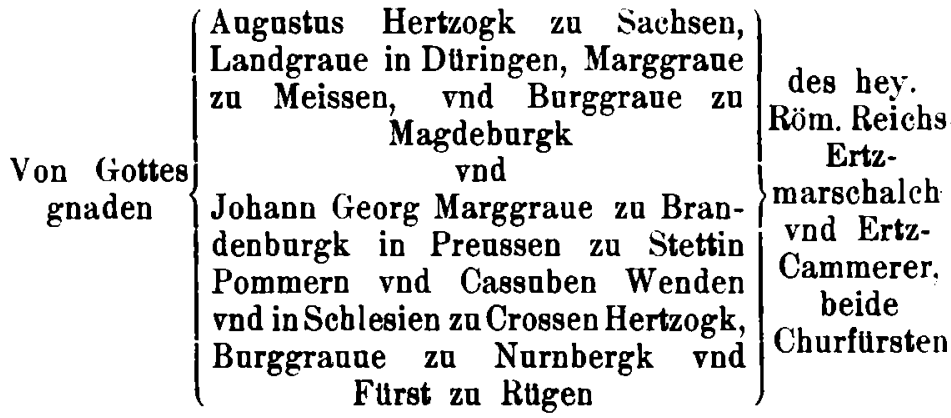

[eigenhändig:] Augustas Charfurst mana propria sabscripsit.

$$
\text { 13. Juli } 1592 .
$$

Herzog Friedrich Wilhelm zu Sachssen ${ }^{1}$ ) an Pfalzgraf Ludwig.

Dem hochgebornen Fursten, vnserm frenndlichen lieben vettern, schwagern, brudern ind geuattern, herren Ludwigen,

1) Vormund Christians II., vgl. Real-Enz..$^{3}$ XI, 87. 
Landgrauen zue Hessen, Granen za Catzenelnbogen, Ditz, Zigenhain rnd Niddaw.

Zu S. L. handen.

[Praes. Map.(') am 23. Julij 1592.]

Inser freundlich dienst vnd was wir liebs rnd gutes vermügen zauorn. Hochgeborner Furst, freandlicher lieber retter, schwager, bruder rnd geuatter, In dieser stunden ist vns von dem schosser zue Stolpen glaubwirdiger bericht einkommen, das vor wenigk tagen der gewesene hofprediger \%ue Dreßden M. Dauid Steinbach zue communiciren begeret, ind als man zu ihme D. Zachariam Rivandrum gelassen, habe er ans eigenem bewegnus seinen biß anhero gefurten Caluinischen irrthumb erkant vnd bekant vnd dartber nicht alleine rnder seiner eigenen handt eine solche Revocation schrifft von sich gegeben, Sondern auch gebeten, dieselbe in der Schloßkirchen za Dreßden offentlichen zuuerlesen, Inmassen $E$. L. ans der beylage ferner zunornebmen. Wie wir nue auber allem zweinell, es werde besage heiliger göttlicher schrifft vber diesen armen buBfertigen sunder im himmel eine große freade furden Engeln Gottes erwecket worden sein, Allso will auch vns menschen in allewege gebaren, rns vber solchem gnadenwerck des heiligen Geistes hertzlichen zuerfrewen, anch hirfur der gottlichen allmacht von hertzen zuloben vnd zuedancken. Der getreue barmbhertzige Gott wolle diesen verlohrnen vnd wieder gefundenen sohn anff der rechten bane seines heiligen alleine seeligkmachenden wortts ferner leitten vnd furen, auch genade verleihen, das alle irrige vnd verfturte zne seines nahmens lob, ehre vnd preib, anch ihrer ewigen seeligkeit nichts wenigers, als mit diesem Steinbach bescheen, zur erkentnus der warheit gefurt vnd gebracht, vnd allso ans den netzen vnd stricken des hellischen jegers gerissen werden mugen, Welches wir ibnen von hertzen wanschen vnd gönnen, Vrid haben E. L. hiruon freundlicher wolmeinunge alsobalden bericht zathuen, nicht vnderlassen wollen, Dero mir angeneme freundliche dienste zuertzeigen geulissen, Datum Mabfeldt am 13. Julij Anno 1592.

Von Gottes genaden Friedrich Wilhelm Hertzog zue Sachssen, vormundt vnd der Chur Sachssen Administrator, Landgraff in Daringen vndt Marggraff zue Meissen.

[eigenhändig:] Friederichwilh z Sachssenn. 